CLINICAL ETHICS

\title{
Are patient information leaflets contributing to informed consent for cataract surgery?
}

\author{
H Brown, M Ramchandani, J T Gillow, M D Tsaloumas
}

J Med Ethics 2004;30:218-220. doi: 10.1136/jme.2003.003723

See end of article for authors' affiliations .....................

Correspondence to: Miss H Brown, The Birmingham and Midland Eye Centre, City Hospital NHS Trust, Western Road, Birmingham, UK; Hbrown@noncom. force9.co.uk

Received 4 March 2003 Revised 30 May 2003 Accepted 1 July 2003

\begin{abstract}
Aim: To assess, against a checklist of specific areas of required information and using standard published criteria, to what extent leaflets given before cataract surgery provided patients with enough information to give adequately informed consent.

Method: Twelve ophthalmology departments in the West Midlands region were asked to submit the cataract information leaflets given to their patients at the preoperative assessment for analysis. Using criteria published by the General Medical Council, British Medical Association, and Medical Defence Union the leaflets were assessed for their contribution to informed consent for patients considering cataract surgery. Leaflets were scored according to the information they provided on: diagnosis, prognosis, treatment options, costs to the patient, details about the procedure, its purpose, likely benefits, how to prepare for it, what to expect during and after the operation, and the common as well as serious complications that may occur. The readability of the information was also assessed.

Results: All the units' leaflets provided information on diagnosis, the lifestyle changes required postoperatively, and cost involved to the patient. Only five units had leaflets that mentioned the risks involved in cataract surgery. The other areas of information were covered by $50-75 \%$ of the leaflets. Fifty per cent of the leaflets included a diagram. The average SMOG readability score was high.

Conclusion: Although present cataract information leaflets make some contribution to the process of informed consent, most do not address important areas outlined by the General Medical Council. Many of the areas of information that are required for informed consent could easily be covered, and should be borne in mind when designing patient information leaflets. Resources are available on the internet including toolkits, guides, and means of assessment for the production of patient information leaflets.
\end{abstract}

$P$ atients must be given sufficient information, in a way they can understand, to enable them to exercise the right (protected in law) to make informed decisions about their care. ${ }^{1}$ The provision of information requires effective communication primarily by discussion. Verbal information is useful if it is provided in a manner intelligible to the hearer and at a pace at which the recipient can digest it. Leaflets allow patients to digest information at their own speed and are a point of reference. Patient information leaflets could therefore provide a valuable contribution to informed consent.

By using standard published criteria we set out to assess whether leaflets for patients considering cataract surgery were contributing to informed consent.

\section{METHOD}

Twelve ophthalmology departments in the West Midlands region of the United Kingdom were asked to submit the cataract information leaflets given to their patients at the preoperative assessment for analysis. Leaflets were compared, and scored against previously set criteria for obtaining consent. The criteria were derived from publications by the General Medical Council (GMC), ${ }^{1}$ British Medical Association (BMA), ${ }^{2}$ and the Medical Defence Union (MDU). ${ }^{3}$ The presentation of the leaflets was also analysed, as was the readability of the material using the "simple measure of gobbledegook" or SMOG readability formula. ${ }^{4}$

\section{RESULTS}

All 12 of the region's ophthalmic units provided their cataract information leaflets. The number of separate leaflets given to a patient varied from one to six. The key areas on which the leaflets were scored are outlined in tables 1 and 2. Table 1 shows how many of the 12 ophthalmic units had cataract information leaflets covering the areas required for informed consent, and table 2 shows the readability of the patient cataract information leaflets.

Of the 12 areas of information listed in table 1, the leaflets reviewed in this study covered an average of 6.9 (range 4-10). All of the units explained the diagnosis of cataract, and nine also gave accounts of the symptoms and causes of a cataract. Although the improvement of vision was implied by all of the leaflets, it was only stated by one. Two units did not describe the procedure of cataract extraction other than stating that the cataract is removed.

Nine of the 12 units mentioned the fact that new glasses were likely to be required after the operation. Seven units stated that review would be required in clinic, with four of these indicating the likely number of follow up visits. Four leaflets commented that some additional treatment might be required at a later date, such as posterior YAG laser capsulotomy and secondary intraocular lens insertion, if problems arose during the initial surgery.

None of the leaflets stated that cataracts are normally harmless if left untreated. Three units had leaflets that mentioned that vision could be lost after cataract extraction. No leaflet mentioned that there was a risk of losing the eye. Seven of the units described signs and symptoms that should prompt the patient to seek help urgently and all provided a contact number on the leaflet.

\section{DISCUSSION}

The GMC has published a leaflet "Seeking patients' consent: the ethical considerations". ${ }^{1}$ In this it is stated that patients have a right to information about their condition and the treatment options available to them. The information that a 
Table 1 Areas of information provided by the 12 ophthalmic units

\begin{tabular}{lc}
\hline Area of information & Units providing information \\
\hline Diagnosis & 12 \\
Treatment options & 8 \\
No treatment option & 1 \\
Purpose of procedures & 1 \\
Details of procedures & 10 \\
How to prepare for the operation & 7 \\
What to expect during procedure & 6 \\
Lifestyle changes required & 12 \\
Costs to patient & 12 \\
Risks & \\
Any & 5 \\
Common & 3 \\
Serious & 3 \\
\hline
\end{tabular}

patient ought to know before consenting to treatment may include: details of the diagnosis; prognosis; treatment options (including no treatment); and costs to the patient. Information about the procedure should include: its purpose; likely benefits; how to prepare for it; what to expect during and after the procedure; and the common as well as serious effects that may occur.

In common with a previous study that looked at patient concerns, the most common fault we found was emphasis of the benefits coupled with little attention given to the risks and side effects. ${ }^{5}$ In our study, only five units mentioned any of the risks of surgery in their written information. In two of the three leaflets that mentioned visual loss, it was described as "very uncommon" or "very rare". None of the leaflets mentioned that there was a risk of losing the eye. Quantitative information about outcome probabilities was lacking, with only two of the units giving a figure for the probability of successful treatment (around 95\%). Two further units indicated that the majority of patients did well, although a figure was not quoted. In one leaflet the only statistic given was that "injuries caused by accidents due to poor vision caused by cataracts were more likely to happen than damage resulting from the complications of cataract surgery".

One unit's information stated that the only treatment for cataract was surgery, and that without surgery the cataract would only get worse and result in the patient not being able to see anything. None of the leaflets stated that cataracts are normally harmless if left untreated. Circumstances in which an operation might be considered (cataract interfering with lifestyle, glasses unable to improve vision or needing to be changed frequently) were covered by six of the units. Although most cataract operations are now carried out by phacoemulsification, the different ways of cataract extraction were mentioned by only one leaflet.

There was much room for improvement in the presentation of material. Although six out of 12 centres included a labelled diagram for ease of explanation, only four of these explained the labelling. The number of leaflets given to a patient at the preoperative assessment varied, with most centres (nine of 12) providing three or more separate leaflets, and one giving six. When more than two leaflets were given, repetition of information was often found. One of the units provided a single 19 page A4 "leaflet". The leaflets were scored for their readability using the SMOG readability formula. The average score was 10 with a range of 7-12. The recommended level for such information leaflets is a score of 5 or lower as this will be understood by most people. ${ }^{6}$ It may be useful to the reader to know that a way of assessing readability is incorporated into most computer software. The leaflets' print type and font size will be of importance, as many will have
Table 2 Presentation of information

\begin{tabular}{ll}
\hline SMOG readability score & Mean 10 (range 7-12) \\
Font size & Range 10-26 \\
Number of leaflets & Mean 2.75 (range 1-6) \\
Diagram included & 6 \\
Explanation of labels & 4 \\
\hline
\end{tabular}

reduced reading vision. The font size of the material provided varied from a near vision reading of N20 to N26 for headings and N10 to N20 for text (newspaper print $=\mathrm{N} 8$ ). The finding of one of the leaflets having a printing error with an unfinished sentence was, we felt, unacceptable. Only one unit provided us with evidence that they had written information available in other languages. In contrast to the findings in Coutler's paper, ${ }^{5}$ most of the information in our study was dated (seven out of 12). Of those that were dated, the average age of the leaflets was 3 years old.

Patients have requested written information, not only to help them understand the problem, but also to make the most of their consultation and legitimise seeking help. ${ }^{5}$

Presentation of information for informed consent should involve up to date written material and visual aids, including drawings and diagrams to explain complex aspects of treatment. ${ }^{13}$ Written material, although not a substitute for appropriate discussion, is the main source of supplementary information and can be designed to cover at least the aforementioned areas. It is useful because of limited consultation times, and our underestimation of patients' desire to cope with information and overestimation of their ability to comprehend verbal information (as discussed in a review article by Thomas et $\mathrm{al}^{7}$ ).

Although in verbal discussion doctors are able to vary the amount of information given as felt to be appropriate, one could argue that there should be as much information available as the patient needs or desires. ${ }^{2}$ This has legal implications, and leaflets also have a role in risk management strategies. Risks have been defined as those to which a reasonable person in the patient's position would be likely to attach significance. In Australia, in the case of Rogers $v$ Whittaker, ${ }^{8}$ a doctor did not inform a patient of a complication of cataract surgery, which was rare but carried serious consequences, when asked by that patient specifically to be told of any such risk. The judge ruled in favour of the patient despite the Bolam principle, which still holds in UK courts (that a reasonably competent practitioner in a similar position would have acted similarly and was supported by a responsible body of relevant professional opinion). ${ }^{9}$

\section{CONCLUSION}

Although cataract information leaflets currently given at the preoperative assessment make some contribution to the process of informed consent, most do not address important issues outlined by the GMC. Labelled diagrams and explanations of terms should be used to aid understanding when designing an information leaflet. Patient information leaflets provide a good opportunity to reinforce the patients' knowledge required for informed consent. Many of the areas of information that are required for informed consent can easily be covered, and should be borne in mind when designing patient information leaflets. There are several resources to help information providers improve the quality of their leaflets. Many of these are available on the Internet-for example, a toolkit for producing information leaflets (www.doh.gov.uk/nhsidentity/toolkit_index.htm) and the DISCERN tool, which is a brief questionnaire providing a valid and reliable way of assessing the quality of written information (www.discern.org.uk). The King's Fund has 
recently published a guide for information providers, "Producing Patient Information: How to research, develop and produce effective information resources" by Mark Dunman. The Help for Health Trust's Centre for Health Information Quality (CHIQ) is another useful resource. Existing leaflets could easily be modified to contribute more to informed consent, and this could be an effective risk management strategy when the financial costs of litigation relating to consent are considered.

\section{Authors' affiliations}

H Brown, M Ramchandani, The Birmingham and Midland Eye Centre, Birmingham, UK

J T Gillow, North Staffordshire Hospital, Stoke on Trent, UK

M D Tsaloumas, Selly Oak Hospital, Birmingham, UK

\section{REFERENCES}

1 General Medical Council. Seeking patients consent: the ethical considerations. GMC 2001.

2 British Medical Association. Medical ethics today. Its practice and philosophy. 1993. See chapter 1 .

3 Medical Defence Union. Consent to treatment.

4 McGraw HC. SMOG Grading: A new readability formula. Adapted from Mclaughin G. J Reading 1969;12:639-46.

5 Coulter A. Sharing decisions with patients: is the information good enough? BMJ 1999;318:318-22.

6 Estey A, Musseau A, Keehn L. Comprehension levels of patients reading health information. Patient Education Counselling 1991;18:165-9 and 1996:30:205-8.

7 Thomas R, Thornton H, Mackay J. Patient information materials in Oncology: Are they needed and do they Work? Clin Oncol 1999;11:225-31.

8 Rogers v Whitaker (1992) 67 AWR 47.

9 Sidaway $v$ Board of Governors of Bethlem Royal and the Maudsley Hospital (1995) 2 WRL 480

10 Bolam v Friern Hospital Management Committee (1957) 1 WRL 582. 